\title{
Bordetella trematum infection: case report and review of previous cases
}

\author{
Thaís Regina y Castro ${ }^{1}$, Roberta Cristina Ruedas Martins², Nara Lúcia Frasson Dal Forno ${ }^{3}$, Luciana Santana ${ }^{4}$, \\ Flávia Rossi ${ }^{4}$, Alexandre Vargas Schwarzbold ${ }^{1}$, Silvia Figueiredo Costa ${ }^{2}$ and Priscila de Arruda Trindade ${ }^{1,5^{*}}$ (D)
}

\begin{abstract}
Background: Bordetella trematum is an infrequent Gram-negative coccobacillus, with a reservoir, pathogenesis, a life cycle and a virulence level which has been poorly elucidated and understood. Related information is scarce due to the low frequency of isolates, so it is important to add data to the literature about this microorganism.

Case presentation: We report a case of a 74-year-old female, who was referred to the hospital, presenting with ulcer and necrosis in both legs. Therapy with piperacillin-tazobactam was started and peripheral artery revascularization was performed. During the surgery, a tissue fragment was collected, where Bordetella trematum, Stenotrophomonas maltophilia, and Enterococcus faecalis were isolated. After surgery, the intubated patient was transferred to the intensive care unit (ICU), using vasoactive drugs through a central venous catheter. Piperacillintazobactam was replaced by meropenem, with vancomycin prescribed for 14 days. Four days later, levofloxacin was added for 24 days, aiming at the isolation of S. maltophilia from the ulcer tissue. The necrotic ulcers evolved without further complications, and the patient's clinical condition improved, leading to temporary withdrawal of vasoactive drugs and extubation. Ultimately, however, the patient's general condition worsened, and she died 58 days after hospital admission.
\end{abstract}

Conclusions: Despite being a rare finding, B. trematum is typically associated with the clinical manifestation of disorders that predispose to ulcer development, which can be infected by microorganisms. The combination of antibiotic therapy and surgical debridement plays a key role in preventing systemic infections. Monitoring the appearance of new cases of $B$. trematum is essential, since it can be an emerging microorganism. Isolating and defining the clinical relevance of unusual bacteria yields a more accurate perspective in the development of new diagnostic tools and allows for assessment of proper antimicrobial therapy.

Keywords: Foot ulcer, diabetic, Bordetella infection, Antimicrobial, Susceptibility breakpoint determination

\section{Background}

Bordetella trematum is an infrequent gram-negative coccobacillus [1], typically related to tissue infections. Related information is scarce due to the low frequency of isolates [2]. As laboratories gain greater access to technologies for accurate and specific bacterial identification, rare microorganisms can arise. It is essential to understand the clinical significance of these unusual findings

\footnotetext{
* Correspondence: priscila.trindade@gmail.com

${ }^{1}$ Universidade Federal de Santa Maria, Santa Maria, Brazil

${ }^{5}$ Departamento de Análises Clínicas e Toxicológicas, Centro de Ciências da

Saúde, Universidade Federal de Santa Maria, Avenida Roraima, 1000, Camobi,

Santa Maria, RS CEP 97105-900, Brazil

Full list of author information is available at the end of the article
}

and the need for treatment. We reviewed the published case reports and will be discussing a new one here.

\section{Case presentation}

A 74-year-old female patient attended the vascular surgery outpatient clinic and was referred to the hospital for revascularization of the distal arteries. She had necrotic ulcers in both legs, worse in the right. She reported pain, signs of local infection and myiasis on the lateral side of the ankle, tendon exposure, edema, and dry skin, but no signs of acute ischemia. Her underlying diseases were difficult to control: systemic arterial hypertension for 20 years; type II diabetes mellitus (DM) for 13 years; hypothyroidism; a stroke 6 years ago, chronic renal failure class IV; peripheral arterial occlusive disease, and

(c) The Author(s). 2019 Open Access This article is distributed under the terms of the Creative Commons Attribution 4.0 International License (http://creativecommons.org/licenses/by/4.0/), which permits unrestricted use, distribution, and 
postmenopausal osteoporosis. The patient referred to previous angioplasty performed 1 year earlier on the lower right leg due to peripheral arterial occlusive disease. Upon hospital admission, several sites of infection other than skin and soft tissue were discarded. Laboratory tests showed a normal leukocyte count and reactive C protein of $3.98 \mathrm{mg} / \mathrm{dL}$ (reference value: $<0.30 \mathrm{mg} / \mathrm{dL}$ ). Empiric treatment with piperacillin-tazobactam (4.5 g IV $6 / 6 \mathrm{~h}$ ) was initiated, which was prescribed for 5 days.

Two days after admission, surgical debridement was performed. Limb amputation was discussed, but rejected by the patient and family members. During the surgery, a fragment of the ulcer tissue was collected and sent to the hospital's microbiology laboratory. In the staining procedure, a few gram-positive cocci and gram-negative bacilli were observed. The specimen was submitted for enrichment in the brain-heart infusion broth for $24 \mathrm{~h} / 37^{\circ} \mathrm{C}$ and later seeded in $5 \%$ sheep blood agar and MacConkey agar, incubated for $37^{\circ} \mathrm{C}$, and presented growth after $24 \mathrm{~h}$. VITEK 2 system (bioMérieux, Marcy l'Etoile, France) identified Enterococcus faecalis, Stenotrophomonas maltophilia, and B. trematum. The isolate was subsequently identified as B. trematum, using VITEK MS (bioMérieux, Marcy l'Etoile, France) and confirmed by $16 \mathrm{~S}$ rRNA gene sequencing with Illumina MiSeq (Illumina, San Diego, CA, USA). The oxidase test was negative. MICs were determined by Sensititre gram-negative MIC plate (Thermo Scientific, Waltham, MA, USA) (Table 1).

After surgery, the intubated patient was transferred to the ICU, using vasoactive drugs through a central venous catheter. Three days later, she presented a worsening clinical condition. Oxacillin-resistant Staphylococcus hominis was isolated from a blood culture drawn through a peripheral vein. Piperacillin-tazoctam was replaced by meropenem (500 mg IV 24/24 h) and vancomycin (1 g IV 24/24 h), prescribed for 14 days. Four days later, levofloxacin $(750 \mathrm{mg}$ IV $24 / 24 \mathrm{~h}$ ) was added for 24 days aiming at S. maltophilia isolated from the ulcer tissue.

The necrotic ulcers evolved without further complication and the patient's clinical condition improved, leading to temporary withdrawal of the vasoactive drugs and extubation. However, the patient's general condition and kidney function worsened, probably due to the severity of her underlying diseases, and she died from sepsis of cutaneous origin 58 days after hospital admission. Autopsy was not performed. Figure 1 shows a timeline of the events.

\section{Discussion and conclusions}

B. trematum was described in 1996, isolated from human wounds and ear infections [1]. Information about its reservoir, life cycle and pathogenesis remain unknown. Regarding virulence, little is known [5]. Typically associated with tissue infections in diabetic patients, and generally occurs in polymicrobial infections, which further complicates its clinical interpretation [2]. In our case, where E. faecalis and $S$. maltophilia were isolated with $B$. trematum in the ulcer, the role of $B$. trematum in the patient's prognosis became unclear. However, as this microorganism was previously reported as a causative agent of bloodstream infection $[4,6$, $8]$, its interpretation and implication in disease was challenging and required integration of clinical, epidemiological, and microbiological issues.

When first described, B. trematum presented the following phenotypic characteristics: non-glucose metabolizer, grown on MacConkey agar, motile, with variable nitrate reduction, catalase and citrate positive, urease, oxidase and lysine decarboxylase negative [1]. Methodologies such as MALDI-TOF MS and VITEK 2 system were efficient in identifying $B$. trematum. However, there are reports that demonstrate problems regarding microorganism misidentification by API 20 NE (bioMérieux, Marcy l'Etoile, France), due to the absence of $B$. trematum in its identification database [2]. In another case, also using API $20 \mathrm{NE}, B$. trematum was misidentified as Achromobacter denitrificans/ Bordetella bronchiseptica. This may have occurred because the nitrate reduction test was variable, and the oxidase test reagent was different from the one used by other researchers [7]. In some cases, the confirmation of the microorganism identification was carried out by $16 \mathrm{~S}$ rRNA gene sequencing $[3-5,7,8]$. In our case, the isolate was correctly identified by the VITEK 2 system and MALDI-TOF MS and confirmed by $16 \mathrm{~S}$ rRNA gene sequencing. Even with credible identification by using routine laboratory instruments, the available literature only reports a few cases, as summarized in Table 1.

There is no standardized methodology by the Clinical Laboratory Standard Institute (CLSI) or the European Committee on Antimicrobial Susceptibility Testing that performs an antimicrobial susceptibility test, specifically for B. trematum. Some authors have used the CLSI manual as an interbreeding source $[2,5-8]$, cited as the MIC interpretative standards for other Non-Enterobacteriaceae and MIC interpretative standards for Enterobacteriaceae, along with the use of antibiotics such as ampicillin and cephalothin.

Analyzing antimicrobial susceptibility tests performed by other authors (Table 1), B. trematum has always shown sensitivity to piperacillin-tazobactam, which was initially used as an empirical therapy. Indeed, for this antimicrobial, we obtained the same MIC $(\leq 8 / 4 \mu \mathrm{g} / \mathrm{mL})$ as reported by Saksena et al. (2015), which may have been a contributing factor to the favorable outcome of the surgical wound. Cefotaxime showed MIC $32 \mu \mathrm{g} / \mathrm{mL}$ in other studies [2, 7], as it is considered to be an intermediate resistant. The MIC in our study $(8 \mu \mathrm{g} / \mathrm{mL})$ was lower than that described by other authors. However, it is not possible to define whether this MIC represents susceptibility. The problems with MIC 


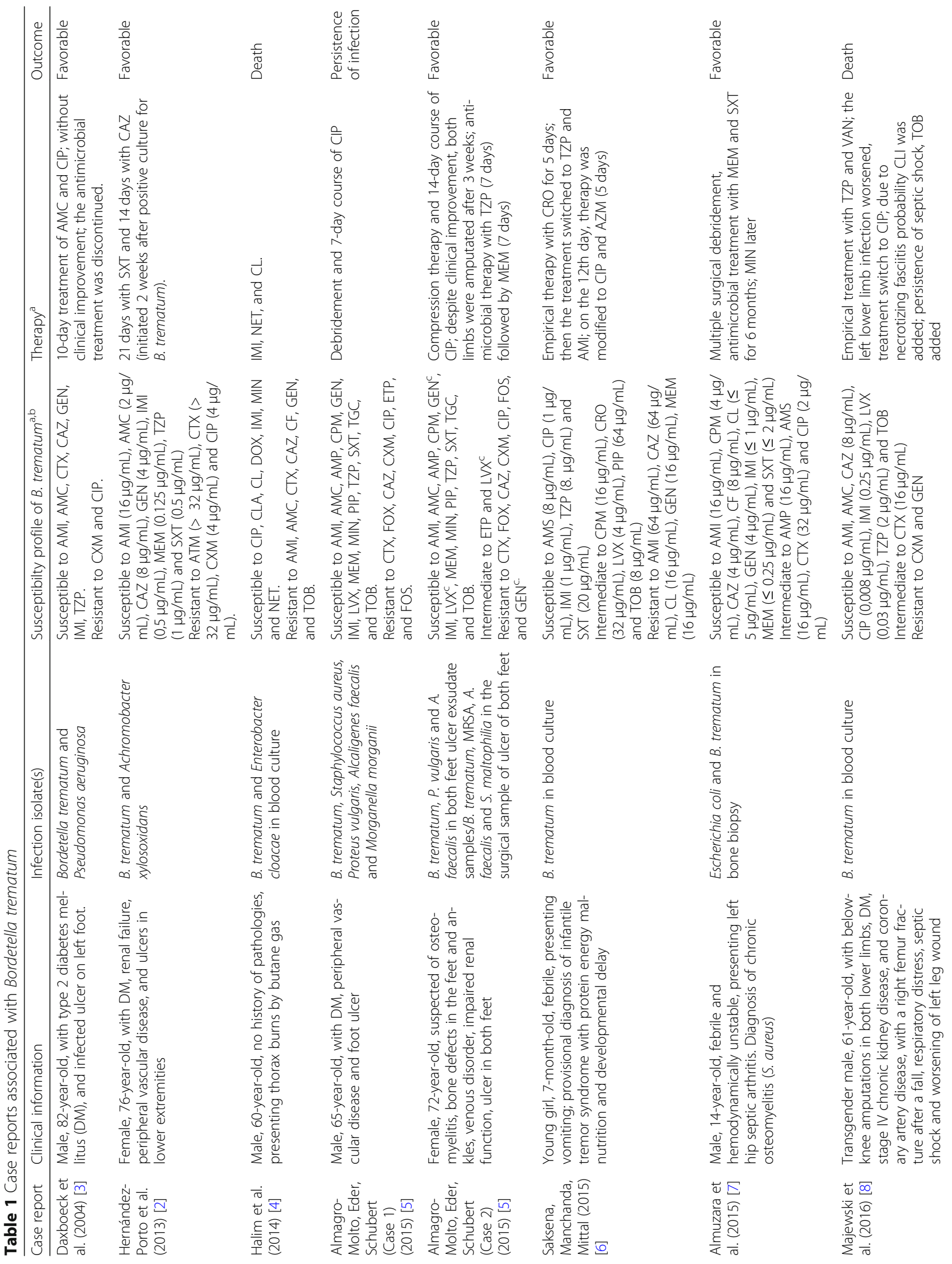




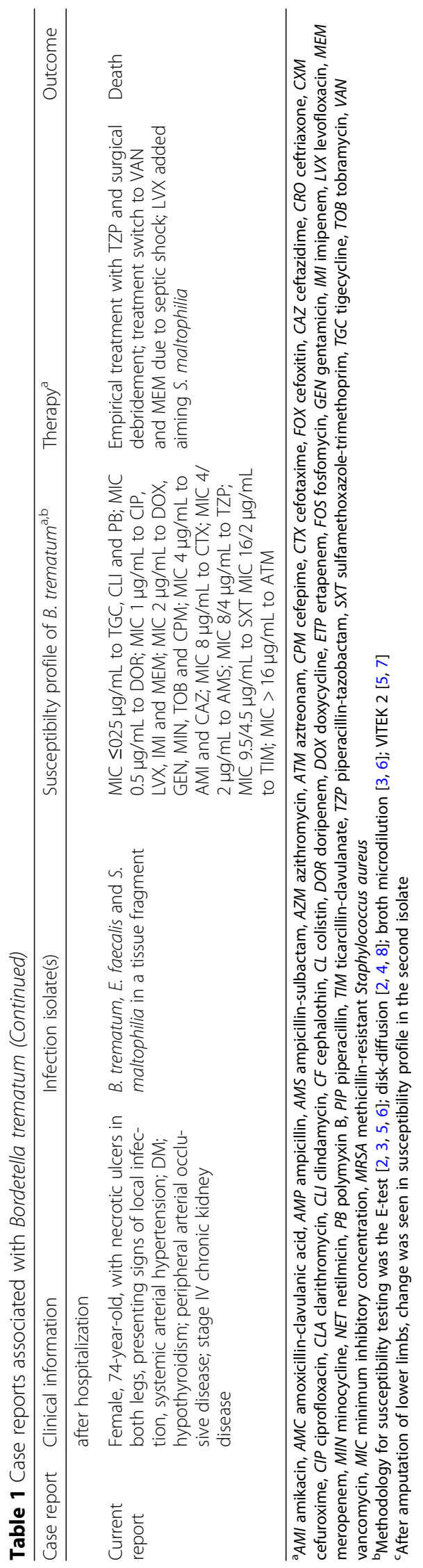






Fig. 1 A timeline of the main events of the patient's illness

interpretation are probably due to lack of in vitro/in vivo susceptibility correlation or breakpoints [6].

Since B. trematum infection/colonization occurs primarily in wounds, debridement is essential for treatment, because it removes pathogens from nonviable tissues and reduces recurrence of the infection. Debridement associated with the correct use of antibiotics can lead to a favorable outcome [5]. However, there were no further cultures to determine whether B. trematum was actually eradicated.

The pathogenicity of this microorganism is not well-established, so it is unclear what the contribution of B. trematum was to the patient's infection and outcome. Despite being a rare finding, $B$. trematum is typically associated with clinical manifestations of disorders that predispose to the development of ulcers that can become infected by microorganisms. The combination of antibiotic therapy and surgical debridement plays a key role in the cure, preventing systemic infections. Monitoring the appearance of new cases of $B$. trematum is essential, as it could be an emerging microorganism. Isolating and defining the clinical relevance of unusual bacteria facilitates an overall perspective towards the development of new diagnostic tools and allows for assessment of proper antimicrobial therapy.

\section{Abbreviations}

CLSI: Clinical Laboratory Standards Institute; MIC: Minimal Inhibitory Concentration

\section{Acknowledgments}

We are grateful to the Microbiology Section of the Laboratório de Análises Clínicas do Hospital Universitário de Santa Maria for the isolation and initial identification of Bordetella trematum. We also thank the Microbiology Laboratory of the Central Laboratory Division at the Hospital das Clínicas da Faculdade de Medicina da Universidade de São Paulo and the Laboratory of Bacteriology LIM54 linked to the Departamento de Doenças Infecciosas e Parasitárias da Faculdade de Medicina da Universidade de São Paulo for confirming the microorganism by MALDI-TOF MS and sequencing the 165 rRNA gene, respectively.

\section{Funding}

Thaís R. y Castro received a Master's degree grant provided by Coordenação de Aperfeiçoamento de Pessoal de Nível Superior (CAPES), Proccess Number 1740547. CAPES is a Governmental Agency that provides grants for graduate students. 


\section{Availability of data and materials}

Data sharing is not applicable to this article, since no datasets were generated or analyzed during the current study.

\section{Authors' contributions}

TC, PAT and AVS were major contributors in writing the manuscript and analyzing the patient records; RCRM and SFC performed $16 \mathrm{~s}$ rRNA sequencing, NLFDF, LS, and FR performed laboratory phenotypic identification of the isolate. All authors have approved the revisions and the submitted version. We confirm that the manuscript has been read and approved by all named authors and that there are no other persons who satisfied the criteria for authorship but are not listed. We further confirm that the order of authors listed in the manuscript has been approved by all of us.

\section{Ethics approval and consent to participate}

The study was reviewed and approved by the National Ethical Committee in Human Research, Brazil (Deliberation number 59563316.6.0000.5346).

\section{Consent for publication}

As the patient had already died, written consent to publish was obtained from her son.

\section{Competing interests}

Dr. Schwarzbold reports grants from Merck Co., Astellas, Gilead, outside of the submitted work. All other authors have no conflict of interest to disclose.

\section{Publisher's Note}

Springer Nature remains neutral with regard to jurisdictional claims in published maps and institutional affiliations.

\section{Author details}

${ }^{1}$ Universidade Federal de Santa Maria, Santa Maria, Brazil. ${ }^{2}$ Faculdade de Medicina da Universidade de São Paulo, São Paulo, Brazil. ${ }^{3}$ Hospital Universitário de Santa Maria, Santa Maria, Brazil. ${ }^{4}$ Hospital das Clínicas da Faculdade de Medicina da Universidade de São Paulo, São Paulo, Brazil. ${ }^{5}$ Departamento de Análises Clínicas e Toxicológicas, Centro de Ciências da Saúde, Universidade Federal de Santa Maria, Avenida Roraima, 1000, Camobi, Santa Maria, RS CEP 97105-900, Brazil.

Received: 9 October 2018 Accepted: 29 April 2019

Published online: 30 May 2019

\section{References}

1. Vandamme P, Heyndrickx M, Vancanneyt M, Hoste B, De Vos P, Falsen E, et al. Bordetella trematum sp. nov., isolated from wounds and ear infections in humans, and reassessment of Alcaligenes denitrificans Rüger and tan 1983. Int J Syst Bacteriol. 1996:46:849-58.

2. Hernández-Porto M, Cuervo M, Miguel-Gómez MA, Delgado T, Lecuona M. Diabetic leg ulcer colonized by Bordetella trematum. Rev Esp Quimioter. 2013;26:72-3.

3. Daxboeck F, Goerzer E, Apfalter P, Nehrt M, Krause R. Isolation of Bordetella trematum from a diabetic leg ulcer. Diabet Med. 2004;21:1247-8.

4. Halim I, Ihbibane F, Belabbes H, Zerouali K, El Mdaghri N. Isolement de Bordetella trematum au décours d'une bactériémie. Ann Biol Clin (Paris). 2014;72:612-4. Available from: https://www.jle.com/fr/revues/abc/e-docs/ isolement_de_bordetella_trematum_au_decours_dune_bacteriemie_ 302630/article.phtml.

5. Almagro-Molto M, Eder W, Schubert S. Bordetella trematum in chronic ulcers: report on two cases and review of the literature. Infection. 2015:43:489-94.

6. Saksena R, Manchanda V, Mittal M. Bordetella trematum bacteremia in an infant: a cause to look for. Indian J Med Microbiol. 2015;33:305-7. Available from: http://www.ijmm.org/article.asp?issn=0255-0857;year=2015;volume= 33;issue $=2 ;$ spage $=305 ;$ epage $=307$; aulast $=$ Saksena .

7. Almuzara M, Barberis C, Traglia G, Sly G, Procopio A, Vilches V, et al. Isolation of Bordetella species from unusual infection sites. JMM case rep. 2015:1-7. Available from: https://jmmcr.microbiologyresearch.org/content/journal/ jmmcr/10.1099/jmmcr.0.000029.

8. Majewski LL, Nogi M, Bankowski MJ, Chung HH. Bordetella trematum sepsis with shock in a diabetic patient with rapidly developing soft tissue infection. Diagn Microbiol Infect Dis. 2016;86:112-4. Available from: https:// www.sciencedirect.com/science/article/abs/pii/S0732889316301572.

Ready to submit your research? Choose BMC and benefit from:

- fast, convenient online submission

- thorough peer review by experienced researchers in your field

- rapid publication on acceptance

- support for research data, including large and complex data types

- gold Open Access which fosters wider collaboration and increased citations

- maximum visibility for your research: over $100 \mathrm{M}$ website views per year

At $\mathrm{BMC}$, research is always in progress.

Learn more biomedcentral.com/submissions 